\title{
Characteristics of patients with drug resistant and drug sensitive tuberculosis in East London between 1984 and 1992
}

\author{
N Al Jarad, S Parastatides, E A Paul, C D Sheldon, H Gaya, R M Rudd, \\ D W Empey
}

\begin{abstract}
Background - The aim of this study was to investigate retrospectively factors associated with drug resistant tuberculosis at the London Chest Hospital.

Methods - The microbiology results for patients with tuberculosis at the hospital for the period 1984-92 were reviewed, together with case notes and chest radiographs of all patients with drug resistant tuberculosis and of 101 patients with drug sensitive tuberculosis notified during the same period as a control group.
\end{abstract}

Results - Culture positive pulmonary tuberculosis occurred in 292 patients. Drug resistant strains were isolated from 20 patients $(6 \cdot 8 \%)$. Ten of the $292(3.4 \%)$ had strains resistant to a single drug and nine $(3 \cdot 1 \%)$ had resistance to more than one first line drug. One patient had strains resistant to isoniazid and capreomycin. Strains resistant to more than one drug were all resistant to isoniazid and rifampicin. In five patients these strains were also resistant to pyrazinamide and in two they were resistant to streptomycin. Single drug resistant strains were resistant to isoniazid (nine patients) or streptomycin (one patient). Among the risk factors studied previous treatment for tuberculosis was the most significant association with drug resistant tuberculosis (7/9) for patients with resistance to more than one drug; 5/11 for single drug resistance compared with $6 / 101$ patients in the drug sensitive group (odds ratio $22 \cdot 8$ ). Other risk factors were bilateral disease at presentation (odds ratio $8 \cdot 5$ ), and disease at a young age (odds ratio 1.03).

Conclusions - Previous treatment for tuberculosis and bilateral disease at presentation were found to be more commonly associated with cases of drug resistant than with drug sensitive tuberculosis.

(Thorax 1994;49:808-810)

The prevalence of drug resistant tuberculosis in the UK is $3-6 \%$ for resistance to one drug and $0.8 \%$ for multiple drug resistance. ${ }^{12}$ These figures are much higher in other parts of the world. The results of culture and sensitivity for mycobacteria usually takes six weeks. Recognition of factors associated with drug resistance to antituberculous treatment on a local basis could result in the use of a more intensive treatment programme and closer observation for those patients who have been recently diagnosed as having tuberculosis and who have risk factors. This also alerts health workers to take greater personal care when dealing with high risk patients. East London has a relatively high rate of notification of tuberculosis of 50-60 per 100000 population (150/100000 for immigrants from Bangladesh) compared with the UK notification rate of 6-10 per 100000 population. ${ }^{34}$

In this study we have identified the characteristics of patients with drug resistant $\mathrm{Myco}$ bacterium tuberculosis seen at the London Chest Hospital between 1984 and 1992 and compared them with a control group of 101 patients with fully sensitive culture positive pulmonary tuberculosis diagnosed within the same period.

\section{Methods}

The case notes and chest radiographs of all patients who were notified as having tuberculosis attending the London Chest Hospital for the period 1984-92 were reviewed, together with the microbiology results of sputum and bronchoalveolar lavage fluid for those with pulmonary tuberculosis. There were insufficient microbiological results from cases of extrapulmonary tuberculosis to justify their inclusion in this study. All specimens were sent to the same laboratory for detection of acid fast bacilli and identification of species and drug sensitivity. Multiple drug resistance was considered to be present when organisms were resistant to more than two first line drugs (isoniazid, rifampicin, streptomycin, pyrazinamide, or ethambutol). The following factors were sought from patients' case notes: (1) date and country of birth; (2) date of entry to UK for foreign born patients; (3) understanding and mastery of the English language; (4) state of employment, accommodation and number of dependents; (5) previous treatment of tuberculosis at any time; (6) family history of tuberculosis; (7) site and extent of the disease on the chest radiograph; (8) other factors such as alcoholism, homelessness, living in hostels or other care homes, illegal substance abuse; and (9) HIV infection.

One hundred and one patients with culture positive pulmonary tuberculosis whose isolates were fully sensitive to all antituberculous drugs were chosen at random as a control group from 272 patients with culture positive pulmonary tuberculosis diagnosed between January 1984 and December 1992. 


\section{STATISTICAL ANALYSIS}

Logistic regression analysis was used to examine the associations between drug resistance and potential risk factors. The dependent variable was resistant or sensitive organisms. Independent variables were age, sex, ethnic origin, extent of the disease (bilateral or unilateral), English (good or poor), alcohol abuse, and homelessness. Complete and reliable data about all these variables were available.

\section{Results}

A total of 506 patients were notified as having tuberculosis between 1984 and 1992 (average 63 cases per year) of whom $428(86 \%)$ had pulmonary tuberculosis. In 292 patients (68\%) sputum or bronchoalveolar lavage fluid grew $M$ tuberculosis.

Isolates of 20 of the 292 patients $(6.8 \%)$ were resistant to one or more first line antituberculous drugs. Of these, $10(3.4 \%)$ had single drug resistance and nine $(3.1 \%)$ had resistance to more than one drug. In one patient strains were resistant to isoniazid and capreomycin. During the analysis this patient was considered as a case of single drug resistance.

Table 1 Demographic data

\begin{tabular}{llll}
\hline & $\begin{array}{l}\text { Resistant to } \\
\text { more than one } \\
\text { first line drug } \\
(n=9)\end{array}$ & $\begin{array}{l}\text { Resistant to } \\
\text { one first line } \\
\text { drug } \\
(n=11)\end{array}$ & $\begin{array}{l}\text { Drug sensitive } \\
\text { tuberculosis } \\
(n=101)\end{array}$ \\
\hline $\begin{array}{l}\text { Male:female } \\
\text { Age: }\end{array} \quad$ Mean & $6 / 3$ & $8 / 3$ & $29 / 72$ \\
$\quad$ Range & 40 & 32 & $47 \cdot 4^{*}$ \\
Caucasian:non-Caucasian & $20-82$ & $16-56$ & $14-78$ \\
\hline
\end{tabular}

* Odds ratio 1.03 (confidence interval 1.02 to 1.05 ), $\mathrm{p}<0.001$.

Table 1 shows that patients with drug resistant tuberculosis were younger than patients with drug sensitive tuberculosis. Patterns of resistance to antituberculous treatment are illustrated in tables 2 and 3. When risk factors were examined the highest risk was associated with previous treatment for tuberculosis with an odds ratio of 22.8 (table 4). Two other factors were bilateral disease at presentation (odds ratio 8.5) and disease at young age (odds ratio 1.03) (tables 1 and 4). Antibody testing for HIV was negative in two patients with drug resistant tuberculosis; no other patients were tested. None of the drug sensitive group were tested for HIV.

All patients with drug resistance were treated with multiple drugs including second line drugs when necessary. None was considered to be suitable for surgical intervention. Two patients with resistance to more than one drug died of tuberculosis after five months and 3.5 years from the time of diagnosis and two went back to their country of origin. Five other patients are still under the care of the hospital for periods ranging from 2 to 12 years. Three patients are still sputum positive and require regular hospital admissions and two became sputum negative after 15 and 26 months of treatment but are still routinely followed up because of bronchiectasis. All patients with single drug resistance were successfully treated and discharged after 12-24 months of follow up.

\section{Discussion}

The $3.8 \%$ prevalence of patients with organisms resistant to more than one drug is four times higher than the national average of 0.5 $0.8 \% .^{12}$ Resistance to rifampicin in patients with multiple drug resistance is particularly worrying. Rifampicin and isoniazid are the two

Table 2 Patients with resistance to more than one first line drug

\begin{tabular}{llllll}
\hline Patient & Age & Sex & Country of origin & Resistant to & Previous treatment \\
\hline 1 & 24 & M & Egypt & INH,R,PZI,ETH & Yes \\
2 & 24 & M & Somalia & INH,R & Yes \\
3 & 30 & F & India & INH,R & Yes \\
4 & 33 & M & Bangladesh & INH,R,S,PZI & Yes \\
5 & 20 & M & Somalia & INH,R,PZI,Ansa,Clof & Yes \\
6 & 39 & M & UK & INH,R,PZI,Cipro & Yes \\
7 & 25 & F & Nigeria & INH,R,Ansa & No \\
8 & 62 & F & UK & INH,R,S & No \\
9 & 82 & M & UK & INH,R,PZI & \\
\hline
\end{tabular}

* Age when resistant strains first detected.

INH = isoniazid; $\mathrm{R}=$ rifampicin; $\mathrm{S}=$ streptomycin; $\mathrm{ETH}=$ ethambutol; PZI = pyrazinamide; Ansa = ansamycin; Clof = clofazimine; Cipro $=$ ciprofloxacin.

Table 3 Patients with resistance to a single first line drug

\begin{tabular}{llllll}
\hline Patient & Age & Sex & Country of origin & Resistant to & Previous treatment \\
\hline 1 & 50 & M & Bangladesh & INH & No \\
2 & 18 & M & Bangladesh & S & No \\
3 & 50 & M & Hong Kong & INH, CAPR & No \\
4 & 16 & B & Bangladesh & INH & No \\
5 & 36 & M & Pakistan & INH & Yes \\
6 & 21 & M & Bangladesh & INH & Yes \\
7 & 23 & F & UK & INH & Yes \\
8 & 26 & F & India & INH & No \\
9 & 56 & M & UK & INH & Yes \\
10 & 75 & M & Jamaica & INH & Yes \\
11 & 41 & F & UK & & \\
\hline
\end{tabular}


Table 4 Prevalence of factors associated with development of drug resistance

\begin{tabular}{|c|c|c|c|c|}
\hline & $\begin{array}{l}\text { Resistant to } \\
\text { more than one } \\
\text { first line drug } \\
(n=9)\end{array}$ & $\begin{array}{l}\text { Resistant to } \\
\text { one first line } \\
\text { drug } \\
(n=11)\end{array}$ & $\begin{array}{l}\text { Drug sensitive } \\
\text { tuberculosis } \\
(n=101)\end{array}$ & $\begin{array}{l}\text { Odds ratio } \\
\text { (confidence interval) }\end{array}$ \\
\hline $\begin{array}{l}\text { Previous treatment } \\
\text { Bilteral disease on presentation } \\
\text { Recent entry to UK for } \\
\text { foreign-born patients } \\
\text { Alcoholism } \\
\text { Psychological disturbances } \\
\text { Homelessness } \\
\text { Living in care homes (hostels, } \\
\text { homes for the elderly) } \\
\text { Foreign birth } \\
\text { Poor understanding of the } \\
\text { English language }\end{array}$ & $\begin{array}{l}7 \\
8 \\
4 / 6 \\
1 \\
1 \\
0 \\
3 \\
3 \\
4\end{array}$ & $\begin{array}{l}5 \\
8 \\
3 / 8 \\
0 \\
0 \\
0 \\
3 \\
3 \\
6\end{array}$ & $\begin{array}{l}6^{* *} \\
36^{*} \\
19 / 47 \\
9 \\
0 \\
0 \\
11 \\
55 \\
29 / 47\end{array}$ & $\begin{array}{l}22.85(5.1 \text { to } 102.5) \\
8.5(2.1 \text { to } 35.0) \\
0.74(0.22 \text { to } 2.44) \\
\text { Unidentified } \\
\text { Unidentified } \\
\text { Unidentified } \\
\\
3.5(0.97 \text { to } 12.6) \\
0.45(0.14 \text { to } 1.4) \\
0.62(0.19 \text { to } 2.02)\end{array}$ \\
\hline
\end{tabular}

${ }^{*} \mathrm{p}<0.005 ;{ }^{* *} \mathrm{p}<0.001$.

most potent drugs for sterilising the tuberculous focus. When resistance to rifampicin occurs in the presence of resistance to isoniazid the prospects of successful chemotherapy are greatly diminished.

In our study, as in others, previous (and presumably inadequate) treatment of tuberculosis was the most important factor associated with drug resistance. ${ }^{5}$ Bilateral disease was commoner in patients with drug resistant tuberculosis than in those with drug sensitive organisms. Bacterial load in patients with extensive disease is high and the likelihood of the development of resistant strains or of the resistance to emerge after erratic intake of medication is high.

Our study has several limitations which are often encountered in retrospective studies. We might have underestimated the numbers of patients who had had previous treatment in both groups. Data about some risk factors particularly recent entry, housing status, employment and number of dependents - were not always recorded in detail. Tests for detection of HIV antibodies were only done in two cases so we cannot assess the relevance of this factor. Our results are consistent with those from other studies, however, and alert us to the particular factors of importance in our local population in predicting the risks of drug resistant tuberculosis.

We conclude that when patients have a history of previous treatment for tuberculosis and present with relapse or reinfection there should be a high suspicion of drug resistance and it might be advisable to treat with an initial regimen including at least two drugs which have not been previously used. There should also be a suspicion of drug resistance when there is bilateral disease. In all these cases close supervision is essential.

1 Warburton ARE, Jenkins PA, Waight PA, Watson JM. Drug resistance in initial isolates of Mycobacterium tuberculosis in England and Wales, 1982-1991. Communicable Disease Report (CDR review) 1993;13:175-9.

2 Medical Research Council Cardiothoracic Epidemiology Group. National survey of notifications of tuberculosis in England and Wales in 1988. Thorax 1992;74:770-5.

3 Communicable disease statistics, England and Wales. Publications of Office of Population and Censuses and Surveys (OPCS) 1990; Series MB2 no.17:7-21.

4 Duckworth G, O'Sullivan J. Notification of infectious diseases 1991 and 1992. Tower Hamlets People No 5. Publications of Department of Health Medicine, 1992/3:59.

5 Friedman TR, Sterling T, Pablos-Mendez, Kilburn JO, Cauthen GM, Dooley SW. The emergence of drug-resistant tuberculosis in New York city. $N$ Engl $f$ Med 1993;328:521-6. 